\title{
Recent Developments on the Elucidation of Colloidal Aspects of Asphaltenes and Their Relevance to Oilfield Problems
}

\author{
Lia B. S. Balestrin ${ }^{\circledR \#, a}$ and Watson Loh ${ }^{\circledR *, a}$ \\ ${ }^{a}$ Instituto de Química, Universidade Estadual de Campinas (Unicamp), \\ CP 6154, 13083-970 Campinas-SP, Brazil
}

\begin{abstract}
Asphaltenes constitute the heavy petroleum fraction responsible for deposition events that may lead to reduced oil production, therefore of great interest for flow assurance. These molecules selfassemble in solutions leading to formation of aggregates that eventually grow towards precipitation and blockages in reservoirs and pipelines. Based on the Yen-Mullins aggregation model, two complementary scenarios are involved in asphaltenes phase behavior: one called thermodynamic, in which interacting molecules and other species can be assumed to be in equilibrium, and a second one, involving interacting colloidal particles, both being described by different theoretical frameworks. For the first, molecular interactions can explain the experimental observations or theoretical models. For the second stage, colloidal forces such as those described by Derjaguin, Landau, Verwey and Overbeek (DLVO) theory, steric particle stabilization and diffusion or reaction limited aggregation processes might control the process. Our evaluation is that this second approach is underrepresented in the current literature. For this reason, this review focuses on describing evidences for the presence of colloidal particles in crude oils obtained with different experimental techniques, drawing attention to this important attribute and we raise a few questions that we believe must be addressed in order to better understand the contributions from colloidal aspects.
\end{abstract}

Keywords: asphaltene, aggregation, colloids

\section{Introduction to Asphaltenes: from Solubility Class to Molecular Details and Their Amphiphilic Nature}

Crude oil has its composition based on paraffinic and aromatic molecules with different contents of sulfur, oxygen and nitrogen. In its composition, there are also small amounts of vanadium, iron, nickel and cooper. ${ }^{1}$ These composition changes with the geophysics and the age of the well. ${ }^{1}$

There are different techniques used in the fractionation of petroleum. SARA analysis is the most commonly used, and the compounds are separated into four fractions (saturates, aromatics, resins and asphaltenes) based on their polarity. There are different methods of separation and the experimental protocol can follow a combination of old and new methods. The separation methods are mainly based on three different techniques: gravity-driven chromatography separation, thin layer chromatography and high pressure chromatography separation. ${ }^{2}$ For example, clay-gel

*e-mail:wloh@unicamp.br

"Present address: Oxiteno S. A., Av. das Indústrias, 365, 09380-903 Mauá-SP, Brazil adsorption chromatography is the method employed by the ASTM D-2007. However, for oils with asphaltenes content greater than $0.1 \mathrm{wt} . \%$, it is necessary to perform the asphaltene removal by using $n$-pentane in a large excess. Following this step, the $n$-pentane soluble fraction is charged into a column containing clay in the upper section and silica gel plus clay in the lower section. The method uses $n$-pentane and toluene-acetone mixture (1:1 in volume) to promote the separation of the three other classes. ${ }^{3}$

The composition of the saturated fraction is mainly of aliphatic compounds. The aromatics contain molecules bearing benzene rings connected to lateral aliphatic chains. Besides, resins are heavier than the saturated and aromatic fractions, ${ }^{1}$ and can be divided into four different categories: with higher aliphatic character, with greater aromatic character, based on dicyclopentadiene or based on styrene. ${ }^{4}$

Finally, the fraction of asphaltenes is the most complex fraction of the petroleum, ${ }^{1}$ indicated as the solid oil. ${ }^{5}$ It presents as solubility characteristic the precipitation upon the addition of some $n$-alkanes. ${ }^{1,6-8}$ The solid obtained can be solubilized in toluene or other solvent such as benzene, carbon disulfide and chloroform. 
These fractions present distinct solubility characteristics, commonly used for their separation. However, the complexity of the chemistry of these fractions is extensive and often a thin line separates some compounds. For example, mass spectrometry analyses have identified more than 100,000 different chemical compounds in petroleum. ${ }^{5}$ In addition, elemental analysis data show the difficulty of comparing fractions, because wells of different origin may exhibit distinct trends. ${ }^{1}$ Nevertheless, the characterization of the respective fraction can employ the concentration of sulfur in a compound, because this element is more prominent in aromatic polynuclear rings than in paraffins chains. Therefore, the saturated fraction presents the lowest sulfur concentration, while the aromatics and asphaltenes fraction present the highest. ${ }^{1}$ Furthermore, from the hydrogen/carbon ratio, one may observe that asphaltenes present a higher aromatic characteristic than resins. ${ }^{9}$

Nuclear magnetic resonance can provide the analytical differentiation of these fractions. In the case of the saturated one, the hydrogen atom occurs predominantly in the methylene form. ${ }^{1}$ The amount of $\mathrm{H} \alpha$, which indicates the proportion of ramifications in the structure, follows an increasing tendency of saturates, aromatics, resins and asphaltenes. ${ }^{1}$ In addition, naphthenic hydrogens are present in asphaltenic and aromatic fractions; and absent in resins and saturates. ${ }^{1}$

Asphaltenes do not have a specific molar mass because of their polydisperse nature. ${ }^{5,6,8,10}$ Currently the most accepted average molar mass in the literature is about $750 \mathrm{~g} \mathrm{~mol}^{-1} .{ }^{11}$ Figure 1 shows the structural and spatial representation of some of the molecules that compose the fraction of asphaltenes. ${ }^{12}$

The content, molar mass distribution and nature of the asphaltenes molecules, as well as the oil, vary according to their origin. In addition, factors such as the flocculation agent, the time used for the precipitation, the process used and the oil/flocculant ratio also influence the physicalchemical characteristics of the solid obtained (typically varying from brown to black coloration). ${ }^{6-8}$

The asphaltenes composition, like petroleum, is basically of carbon and hydrogen atoms, with smaller amounts of nitrogen, oxygen, sulfur and some transition metals. However, there is still a great deal of questioning about the definition of their chemical structure. ${ }^{7}$ It is still discussed whether asphaltenes molecules adopt the configuration of island (with only a polycyclic structure) $)^{5}$ or archipelago (with more than one polycyclic structure; heteroaromatic molecules connected by short saturated alkyl chains and surrounded by short lateral alkyl chains). ${ }^{13}$ This distinction in molecular architecture remains one of the most controversial issues in petroleum chemistry. However,
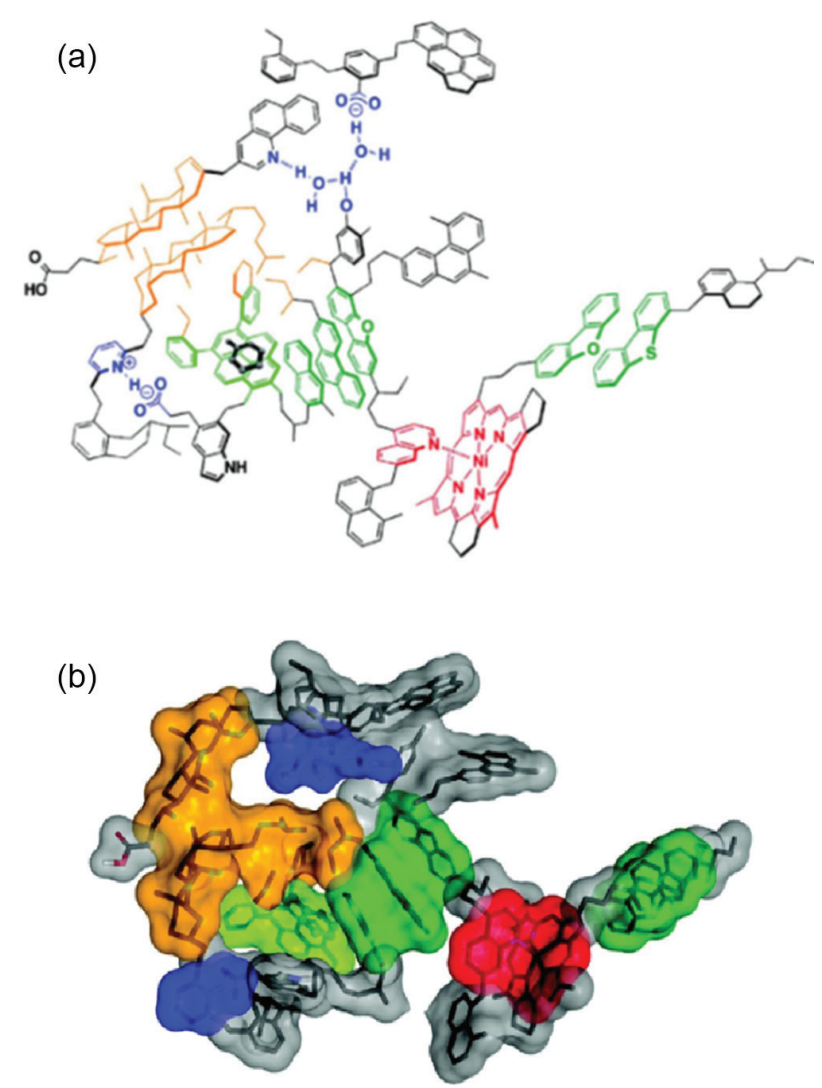

Figure 1. (a) Structural and (b) spatial representation of some of the molecules that compose the asphaltenes fraction, interacting with each other (reproduced from reference 12 with copyright permission 2011 from American Chemical Society).

the asphaltenes pyrolysis product indicates the coexistence of continent-type structures and archipelago. ${ }^{14}$

Figure 1 also shows that the asphaltenes display polar and apolar groups, associated mainly to aromatic and aliphatic groups, respectively. Besides, it shows the possibility of different molecules interacting, leading to the formation of aggregates. The main interactions observed in these aggregates are of the $\pi-\pi$ type. ${ }^{7,12}$ However interactions such as Brønsted's acid-base, ${ }^{12,15}$ hydrogen bonding, ${ }^{12,16}$ polar interactions, ${ }^{17}$ metal coordination, ${ }^{12}$ interactions between cycloalkyl and alkyl ${ }^{12}$ groups forming hydrophobic pockets and resin interactions ${ }^{18}$ may lead to stabilization of the aggregates, affecting the flocculation and/or precipitation of the system.

\section{Phenomena Associated with Asphaltenes in Petroleum Exploration and Production}

Because of their characteristics of aggregating and clogging pipes, asphaltenes are metaphorically regarded as "petroleum cholesterol". ${ }^{19}$ In this sense, aggregation can lead to deposition during oil extraction, which can damage equipment as well as clog installations and pores of the 
rocks. Consequently, asphaltenes deposition can significantly decrease the rate of oil production by interrupting continuous production. Such an event is extremely undesirable in the current economic context, where fossil fuels meet the largest share of energy demand in the world. In addition, energy consumption growth of $56 \%$ is forecast between 2010 and 2040 (US Energy Information Administration). ${ }^{10}$ This scenario requires the oil industry to meet the challenge of producing oil under unconventional and complex conditions, such as in deep water and difficult-to-access formations. ${ }^{10}$

Asphaltenes precipitation is therefore a barrier in this scenario and finding ways to avoid and/or minimize it becomes mandatory in order to reduce the risk of abandonment of wells and minimize cleanup costs that can reach up to several million US dollars per annum. To illustrate this situation, in the Gulf of Mexico, the average expenses related to asphaltenes deposition are about seventy million US dollars per well when it is necessary to stop production. The losses related to the shutdown can reach five hundred thousand dollars per day, based on a production of ten thousand barrels per day and a price of a barrel of oil of fifty dollars. If the well is lost, the execution of a lateral path can generate a cost of one hundred and fifty million US dollars. ${ }^{10}$

Another problem concerning asphaltenes is their characteristic of stabilizing the water-oil interface, especially near the point of asphaltenes precipitation. ${ }^{17}$ Controlling the stability of these emulsions is crucial especially in the oil transport and production stages, and is also one of the greatest challenges in this segment.

Therefore, the oil industry searches for asphaltenes inhibitors, which may act by different mechanisms in order to prevent aggregation of asphaltenes or break down already formed structures (dispersing or dissolving them). In this sense, the proposition of molecules searches for structures that present acid-base interactions and/or involve steric stabilization..$^{20,21}$

Based on the selection of inhibition considering steric stabilization, as early as 1994 Chang and Fogler ${ }^{15}$ indicated that the length and shape of the alkyl chain is very important for the additive efficiency, being similar steric interference for the aggregate growth.

\section{The Asphaltenes Aggregation Model: Thermodynamic and Colloidal Aspects}

The previous section evidenced the need to understand the key aspects related to asphaltenes aggregation. One can view this process from two approaches: a molecular onebased on specific interactions considering the solubility of the species; and the colloidal one. ${ }^{22}$
In the first approach, the assumption is that precipitation results from decreased solubility of dissolved asphaltenes in crude oil. In the second approach, asphaltenes are already present as colloidal particles that can aggregate leading to precipitation. ${ }^{22}$ The great difference between these two approaches can be considered the structure of interest itself: in the first one, the focus is directly on the molecules; while in the second one the process initiates from molecular aggregates (colloidal particles), with flocculation being the result of the alteration of the composition and/or balance of particle interactions. ${ }^{22}$

Solubility models are based on the concept of solubility parameter (e.g., Hildebrand and Hansen), and assume the oil as being composed by asphaltene and maltene (deasphaltened oil). The properties of the maltene are, in general, calculated using cubic equations of state. Furthermore, changes in the solubility parameter of either phase may disrupt the equilibrium condition and eventually lead to precipitation. ${ }^{22}$ Besides, the difference in asphaltenes composition should bring different solubility parameters, because there will be a difference in the balance of intermolecular interactions. ${ }^{23}$

The Flory-Huggins theory, originally developed for polymer solutions, is widely used to describe the precipitation of asphaltenes. In addition, the FloryHuggins-Zuo theory incorporates the effect of gravity action, resulting in asphaltenes gradient models based on its composition. ${ }^{24}$

In 1995, Rogel ${ }^{25}$ began her studies on aggregation of asphaltenes from computational models, using a molecular approach. The research involved the study of the variation of the asphaltenes solubility parameter according to their state of aggregation. The simulation involved the choice of two asphaltenes model molecules and their aggregates, using atomically detailed models and molecular dynamics simulations to determine the respective Hildebrand solubility parameters. The results agree with experimental evidence suggesting an increase in the number of asphaltenes molecules in the aggregates as the solvent solubility parameter decreases.

In 2002, Rogel ${ }^{26}$ continued to work with thermodynamic models, but experimental results indicated that the first stage of asphaltenes precipitation was due to their tendency to self-organization, forming small aggregates or pseudomicelles. The starting point of the proposed model considers that asphaltenes aggregates are composed of an aromatic nucleus surrounded by aliphatic chains. It also considers the driving force of aggregation as the $\pi-\pi$ interactions between the aromatic nuclei of asphaltenes molecules. In addition, the model considers the spatial limitation given by the steric repulsion of the aliphatic chains that surround 
the aromatic nucleus of asphaltenes aggregates. The model also involves effects of solvent properties and asphaltenes properties on aggregation, as well as interfacial effects. This model makes the following contributions to the aggregation of asphaltenes: transfer of the aromatic part of the solvent to the aromatic nuclei, mixing the aliphatic chains with the solvent, deformation of the aliphatic chains, steric interactions between aliphatic chains and the formation of an aggregate-solvent interface. The molecular modeling reported there does not require the use of information derived from experiments on asphaltenes solutions and can be employed to predict critical micellar concentration values $(\mathrm{cmc})$ and aggregate size. For example, indicating that the greater and more condensed the aromatic structure of the asphaltenes, the smaller the value of the $\mathrm{cmc}$ and the greater the aggregation number.

Later, Rogel ${ }^{27}$ also studied the effect of the formation of mixed aggregates between asphaltenes and resins. She considered that the energy involved in the aggregation arises from the energy balance of the mixed aggregate and the free species (resins and asphaltenes). As a contribution to this balance, the model takes the energy related to the transfer of the aromatic part of the fractions from the medium to the aromatic nuclei in the aggregate, weighted by the solubility parameters and by the solubility of the species. In addition, it considers the energy required to form an interface, as well as the steric repulsion of the aliphatic chains.

The great advantage of molecular models is to address the complex problem of asphaltenes stability in terms of solute-solvent interactions at the molecular level, through solubility parameters. Thus, from a set of tabulated data it is possible to infer whether a medium is compatible with a given asphaltene structure. It is also possible to employ, for example, solvatochromic parameters and solubility parameters to create solubility scales between asphaltenes and solvents. ${ }^{28}$

However, molecular models are restricted to predictions at the molecular level. In addition, predictions from this approach consider the dispersant to be a uniform material. ${ }^{29}$ In situations of asphaltenes flocculation that can lead to complete precipitation and separation, the asphaltenes are no longer in equilibrium with the solution. Moreover, their association occurs in cascade on the colloidal scale, and it is also necessary to consider the dynamics of the process. ${ }^{30}$

Colloidal theories connect the rate of aggregation with interparticle interactions (associated with their size and surface charge).$^{30}$ The literature generally attributes the stability of asphaltenes to steric hindrance, but there are results indicating a major role of electrostatic repulsion in some cases, even in medium of low dielectric constant. ${ }^{30}$

The colloidal approximation has already described the deposition of asphaltenes in pores. For this, it was considered that the process is governed by convection (forces exerted by the viscous fluid in the particle) and by diffusion (Brownian motion). Moreover, it is necessary to consider the affinity between particle and surface, from data on diffusion, surface-particle interactions and hydrodynamic interactions. ${ }^{31}$

Until the 1930s, the focus of the study of asphaltenes remained on the understanding of the molecular structure of asphaltenes. However, the discovery of asphaltenes colloidal behavior indicated the need to seek information beyond their molar mass. ${ }^{32}$ There are three classical colloidal models of asphaltenes stability in petroleum.

In the 1920's, Nellensteyn ${ }^{33}$ developed the first one. In his model, asphaltenes are composed of hydrocarbons of high molecular mass dispersed in the oil, forming a thermodynamically stable colloidal system (micelles). In such systems, precipitation can occur by reducing the solvation power of the oil.

Prior to this, asphaltenes were regarded as a final product of polymer origin from a geological transformation. From Nellensteyn's observations, this view was overcome, explaining that the association of individual molecules on a colloidal scale mimicked the behavior of polymeric materials. ${ }^{34}$ In 1938, Nellensteyn showed the first evidence, from X-ray diffraction experiments, that the precipitated asphaltenes had a graphite-like character, i.e., molecules stacking in layers. This finding suggested that the structure of asphaltenes aggregates display stacked aromatic nuclei. However, the study did not reveal whether this aggregate was a result of the association of small molecules or the folding of a larger molecule by intramolecular interactions. ${ }^{34}$

A second model was proposed by Pfeiffer and Saal in $1940 .{ }^{35}$ According to it, asphaltenes were considered as intrinsically insoluble compounds, which were kept dispersed by the peptizing (i.e., avoiding coagulation) action of resins. Thus, resins desorption from the asphaltenes surface results in precipitation/flocculation. The model also considers that asphaltenes are present in the oil as a particle composed of a core containing greater aromatic character. This nucleus is surrounded by molecules with greater aliphatic character in a gradual transition between the dispersed phase and the oil. Their model was based on rheological measurements, considering the transitions between a dispersed asphaltenes system (sol) for a system of flocculated asphaltenes (gel). For this, the authors performed measurements of deformation under constant tension and elastic recovery for two asphaltenes of different origin. ${ }^{35}$

Subsequent studies have shown that resin content is crucial in determining the stability of the system. For example, at very low resin concentrations, asphaltenes are 
only partially coated. Thus, a resin unit can create a bridge between two distinct asphaltenes particles, leading later to their flocculation. ${ }^{36}$

The third model was proposed by Dickie and Yen ${ }^{37}$ in 1967 and suggests the formation of micelles in a hierarchical manner. Aggregation is treated systematically in its different stages. ${ }^{32}$ In addition, the model joins Nellensteyn's colloidal concept and Pfeiffer and Saal's ${ }^{35}$ model to the concept of self-association, being supported until today by different experimental evidences in macro and microscopic scale. ${ }^{32}$

Previous X-ray diffraction studies performed by Yen et al. ${ }^{38}$ already showed the trend of formation of asphaltenes clusters, indicating the structure of the aggregates as stacked sheets with the nucleus containing four to six aromatic rings (totaling an extension of 8.5 to $15 \AA$ ), distant from each other by approximately $3.6 \AA$. In this model,${ }^{37}$ stacking of aliphatic molecules, resins and asphaltenes would be thought to generate particles. At a higher aggregation level, asphaltenes and resins would give rise to mixed micelles. The authors plotted in their scheme some possible combinations, indicating that specific characteristics could vary between the fractions, particularly in the association with resins. They also indicated the possibility of intercluster association, as well as the formation of particles and micelles. The researchers further pointed out that metals could aid in aggregation. The Yen and Dickie ${ }^{37}$ model was based on measurements of diffraction and X-ray scattering, mass spectrometry, gel permeation chromatography, ultracentrifugation measurements and electron microscopy.

However, some details of the interactions may present some misunderstandings or disagreements considering only the Yen and Dickie ${ }^{37}$ model. For example, the extent of order of the structures must be greater. In addition, a review of reported values for distances between aromatic nuclei should be considered. ${ }^{34}$

An update of this model gave rise to the Yen-Mullins ${ }^{11}$ model with the formation of nanoaggregates, which extend their self-organization to clusters, with an aggregation number equal to the micelles originally proposed by Yen and Dickie. ${ }^{37}$ The model specifies the dominant molecular structure as well as the colloidal aspect of the aggregates. ${ }^{5,39}$ However, this model does not take the participation of resins into specific account, although this is verified in several studies. ${ }^{27,40}$ Figure 2 summarizes the Yen-Mullins ${ }^{11}$ model.

The model considers the aggregation resulting from the balance of attractive intermolecular forces, attributed to $\pi-\pi$ interactions; and repulsive ones, fruit of the steric hindrance of peripheral aliphatic branches. Studies based on small angle X-ray scattering (SAXS) and small-angle neutron scattering (SANS) measurements have revealed an organization of structures compatible with the Yen-Mullins model. ${ }^{11,41-45}$ The model further describes the asphaltenes molecules as a multi-ring aromatic core surrounded by alkyl chains of different extents. ${ }^{43}$

One of the main criticisms that the Yen-Mullins model ${ }^{11}$ receives is the discretization of the structures, considering narrow-sized bands to describe them. In addition, the model initially considered only $\pi-\pi$ interactions as responsible for aggregation. Gray et al. ${ }^{12}$ stated that the sizes of the aggregates available in the literature vary from 2 to $20 \mathrm{~nm}$. The same authors also pointed that other interactions, such as hydrogen bonding, are important for aggregation of structures. ${ }^{12}$ These authors ${ }^{12}$ further pointed out that aggregation can be thought as polymerization (model of Agrawala and Yarranton) $)^{46}$ with the incorporation of adhered compounds.

In addition, studies show that a portion of asphaltenes does not aggregate densely, and is even described as adsorbed resin. Thus, a question that naturally arises is the fact that the $\pi-\pi$ interaction results in the longitudinal alignment of the dense-form structure. As a consequence, other interactions, such as hydrogen bonding, acid-base pairs and other polar interactions, must be considered, leading to more open structures. ${ }^{47}$

Bohne and co-workers ${ }^{48}$ in their fluorescence studies, showed that the type of interaction found is inconsistent with the aggregation model proposed by Yen-Mullins, ${ }^{11}$

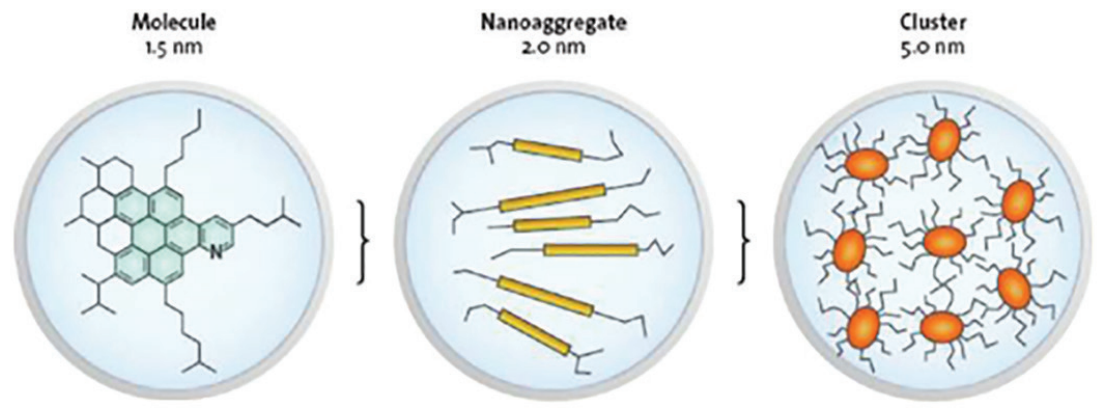

Figure 2. Yen-Mullins model of asphaltenes aggregation (reproduced from reference 11 with copyright permission 2012 from American Chemical Society). 
dependent on the formation of nanoaggregates. Their studies regard this model as simplistic, indicating that considering the driving force of aggregation as the $\pi-\pi$ interaction ignores other important facts. This is because this type of interaction would not be emissive in the fluorescence technique. As signals were detected, these interactions neglected by Yen are probably important in the definition of the aggregation state.

In addition, a more refined view of asphaltenes nanoaggregates containing stacked aromatic nuclei would be needed, with some structure attached at the end to prevent distortion of the aromatic nuclei by steric interference of alkyl substituents. In this sense, there are theoretical studies that have shown that the way in which the cores are stacked may be distant from the initially proposed (vertical), and may include, for example, aromatic nuclei perpendicular to the stack. ${ }^{49}$

Finally, it is necessary to consider the aggregate-solvent interface energy, influenced by the number of aggregation. This is a balance of the geometry of molecules, their solubility and polarizability, as well as their capacity to organize into larger structures. ${ }^{50}$ The representation of asphaltenes aggregates based on Yen-Mullins model ${ }^{11}$ presents inaccuracies and the structure would be unstable by ignoring these important interactions. At present, the exact strength that keeps the larger aggregates stable is unknown, and is generally associated with van der Waals interactions. ${ }^{34}$

\section{Relevant (Emphasis on Recent) Experimental Evidences Supporting the Colloidal Nature of Asphaltenes in Model Systems and in Crude Oils}

Some scientists invested time and a sophisticated methodology of atomic force microscopy (AFM) to provide a molecular description of asphaltenes. The methodology is based on special conditions, like a substrate of copper monocrystal (111) partially covered with islands of two monolayers of sodium chloride, a functionalized tip prepared with a focused ion beam, in order to obtain a single molecule of carbon monoxide in the tip, measurements conducted at approximately $5 \mathrm{~K}$ under ultra-high vacuum (about $1 \times 10^{-13}$ bar). ${ }^{39}$ Recently, a very similar methodology was employed to identify heteroatoms, a challenge considering the contrast of different elements in non-contact atomic force microscopy. The authors ${ }^{51}$ could identify various features (atom and bond contrast, bonding geometry, electron density, substrate interaction) that can be employed to find common heteroatoms ( $\mathrm{S}$ and $\mathrm{N}$ ) and discriminated them from carbon atoms.
However, experimental reports already endorse the presence of asphaltenes as aggregates in crude oil and in solvents such as toluene. A recent article ${ }^{52}$ indicated that asphaltenes colloids may not be the result of aggregation of smaller molecular species but fragmentation of the continuous "skeleton" (a highly cross-linked network of aliphatic, naphtenic, aromatic, asphaltenic, etc blocks). In particular, it was proposed that the crude oil aging can promote the formation of branched mass fractal asphaltenes clusters (macromolecular globules organized in multilevels), related with the increase in the potential instability due to asphaltenes precipitation. ${ }^{52}$ Thus, the effort in complete molecular elucidation should not necessarily result in the elucidation of practical problems such as the choice of precipitation inhibitors. In order to better illustrate this point, several experiments addressing the colloidal behavior of asphaltenes will be described below.

Hoepfner and Fogler ${ }^{41}$ reported SANS studies on asphaltenes solutions in different solvents, identifying asphaltenes clusters like fractal aggregates with sizes within 2-9 $\mathrm{nm}$. They also studied crude oil samples and, in this case, reported objects with dimensions of a few $\mathrm{nm}$ that increase in size as more flocculant ( $n$-heptane) is added or as time progresses. ${ }^{42}$ Barré and coworkers ${ }^{43}$ studied asphaltene aggregates in dilute toluene solutions (a good solvent for asphaltenes) by SAXS and SANS observing disc-shaped aggregates around $3 \mathrm{~nm}$ (radius) and $7 \mathrm{~nm}$ (height). Sirota ${ }^{44}$ also reports SAXS and SANS studies on asphaltenes solutions. He interprets the scattering results as arising from colloidal aggregates generated by concentration fluctuations that exhibit a fractal morphology and solid nature.

In addition to the asphaltenes aggregates characterization, their kinetic studies indicate that the process of growth of structures occurs even in crude oil. However, these studies have been neglected for a long time, being limited to short time scales. Kinetic studies of asphaltenes in crude oils with the addition of alkanes from centrifugation and microscopy techniques have shown that the precipitation time can vary from a few minutes to several months, depending on the alkane (flocculant) concentration employed..$^{53}$ The authors suggest that the addition of a precipitant leads to destabilization of the aggregates already dispersed in the oil by altering their properties. ${ }^{53}$ They also indicate that the proposition of a critical concentration for precipitation (onset) is, therefore, not a unique parameter. In addition, its determination can be treated as an experimental artifact by involving small-scale experiments as a function of time, ${ }^{53}$ because aggregates already exist in the oil and their size increment can be measured if waiting longer.

Recently, the same group organized a critical review about this kinetics question related to the flocculation 
of asphaltenes..$^{54}$ The authors also proposed a model for the growth of the aggregates based on the initial nucleus being already of colloidal nature. It was observed that the model fits adequately to the experimental data, as well as that the collision efficiency becomes greater at higher $n$-heptane concentrations. Thus, shorter times are required for precipitation to start or be detected. ${ }^{54}$

Another relevant contribution to this issue was made by Abudu and Goual ${ }^{55}$ who carried out measurements in systems diluted with $n$-alkanes and toluene in order to characterize the interaction between the oil and solid surfaces under flow conditions. These authors observed that the adsorbed asphaltenes film becomes more viscoelastic as the $n$-alkane concentration approaches the onset concentration. On the other hand, in systems containing toluene as solvent, rigid films were obtained, with a thickness of $3.5 \mathrm{~nm}$, regardless of the asphaltenes concentration. The film formation in systems indicates the existence of dispersed particles, because the system is not exposed to evaporation. Therefore, they propose that the film deposition is due to the juxtaposition of aggregates (first level of aggregation) which interact with the surface from random collisions. In the case where a flocculant is employed, there are aggregates at different stages of growth. This polydispersity may confer the observed viscoelasticity to the generated film.

Wang and co-workers ${ }^{56}$ also evaluated the thickness of the asphaltenes films, but evaluated the effect of adding an electrolyte. The authors showed that electrostatic forces exert a great effect on film organization due to interactions of oil/water and water/solid charged interfaces. ${ }^{56}$ In addition, the authors studied the effect of salinity on different ranges, evaluating their effect on film organization. ${ }^{56}$ For example, at concentrations of 1 to $10 \mathrm{mM} \mathrm{NaCl}$, the surface has hydrophilic characteristics. While, between 10 and $1000 \mathrm{mM}$, the thickness of the film gradually increases, with the surface becoming hydrophobic. ${ }^{56}$ This result indicates that the force structure should be considered for analysis, and the data showed an increase in adhesive forces in the range of 10 to $1000 \mathrm{mM} \mathrm{NaCl}$. In addition, the authors used the Derjaguin, Landau, Verwey and Overbeek (DLVO) theory considering the sum of forces, thus including structural forces in addition to van der Waals interaction and electrostatic forces. ${ }^{56}$ As a conclusion, these authors found a correlation between the experimental data and the calculated curves based on the DLVO model, indicating the importance of treating the colloidal asphaltenes aggregate system. $^{56}$

Zahabi et al. ${ }^{57}$ reported kinetic measurements in a model system, with $16 \mathrm{~h}$ of data acquisition, showing that a steady state is not reached during this period. In addition, the authors showed that asphaltenes deposition occurs in multilayer structures, with a possible structure change or deposition of nanoaggregates. Moreover, the authors have shown that the deposition of asphaltenes depends on their extraction procedure. The authors worked with different asphaltenic fractions, one extracted with $n$-pentane and another with $n$-heptane. Their study showed that deposition is greater for asphaltenes extracted with $n$-pentane, indicating that polydispersity plays an important role in the deposition on the surface of the quartz crystal. The authors also evaluated the effect of the coating of the crystal with carbon steel, gold and iron oxide. In the case of gold, the lowest deposition was observed. While with the carbon steel coating, the deposition was maximum. These observations corroborate with what has already been reported and still reinforce the idea of colloidal particles, since surface characteristics must be mandatory, excluding directional interactions.

In addition, recent results from ultra-small angle X-ray scattering (USAXS) measurements reported by Hoepfner and co-workers, ${ }^{58}$ are capable of accessing the size range of $1 \mathrm{~nm}$ to $5 \mu \mathrm{m}$, therefore providing a full account of asphaltenes particles. Their results describe clusters of soluble and insoluble asphaltenes (following aggregation stages), abandoning the molecular description in organic medium. In addition, it reports that inhibitors display an effect only in the second stage of aggregation, where the objects are in the colloidal range of dimensions. This result shows that the selection of inhibitors could not be solely associated with solubility parameter, which visualizes the actors as molecules. A step forward is necessary, identifying the three-dimensional aggregates and the interactions with their surface. An interesting analogy here can be made with deoxyribonucleic acid (DNA) sequencing: knowledge of the nucleotide sequence does not fully synthesize the information it carries; it is necessary to know its tertiary structure. For asphaltenes, it is not necessary to know the integrality of their molecules, if not all of them will be on the surface of the colloidal particle.

The authors of this review also contributed with experimental evidence to the colloidal view of asphaltenes. We will comment some results that describe this vision.

Systematic SAXS investigation of crude oils in the presence of solvents, flocculants and additives was performed and the results indicated that asphaltenes can be described by the Beaucage fractal aggregation model ${ }^{59}$ with two levels of association. The first level (blue line in Figure 3) has the parameters $\mathrm{R}_{\mathrm{g} 1}$ varying from 2.5 to $5.0 \mathrm{~nm}$ and $\mathrm{P}_{1}$ between 1 and 3 . In parallel, the second level is larger than $90 \mathrm{~nm}$. Therefore, the model based on these results (Figure 3c) describes the aggregates as a fractal 
nanostructure (of exponent $P_{1}$ and radius of rotation $R_{\mathrm{g} 1}$ ) that is arranged in a structure of $R_{\mathrm{g} 2}$ larger than $90 \mathrm{~nm}^{45}$

On the other hand, Figures $3 a$ and $3 b$ (circumferences in red) further show the data of the respective C5-maltenes, which do not comply with this two-level model. The scattering profile undergoes a significant change with the reduction of the scattering intensity, probably due to the extraction of the asphaltenes, the major scattering objects. However, a fraction of soluble asphaltenes (already in the aggregate form) must remain in the maltene samples, leading to an asymmetric background in the high range of scattering vector q. Furthermore, in the region of low $\mathbf{q}$, a power law still persists, indicating the presence of objects larger than $90 \mathrm{~nm}$ (but with lower intensity). The data confirm the removal of a significant fraction of the scattering objects, identified as a fraction of insoluble asphaltenes. These data also suggest that the objects that persist have the same origin as that of their respective oil. ${ }^{45}$

Figure 4 evaluates the effect of good and bad solvents (toluene and $n$-heptane, respectively) on the microstructure of asphaltenes aggregates. According to these data, significant changes occur with the addition of a bad solvent. In this case, there is a greater destabilization of the asphaltenes, resulting in a larger number of molecules in the nanoaggregate and, consequently, the increase in the size of the organizational structure of the first level. On the other hand, toluene does not affect the nanoaggregate structure, but colloidal structures resist even upon dilution of the medium.

In addition, OF3 oil data can be explored in more detail. In this case, the scattering data were obtained exclusively in liquid sample holder, which allows to keep the sample volume constant and to compare the $\mathrm{G}$ factor of the data fitting. An increase of the factor $G$ represents an increase in the number of scattering objects. Figure 5 shows this tendency as an increase in the number of objects upon increasing the concentration of $n$-heptane, as expected for a flocculant. On the other hand, the addition of toluene reduces the number of objects, indicating the partial solubilization of the objects by the action of the solvent. These observations indicate that the aggregates grow as more flocculant is added in the system. Additionally, there are strong evidences of a good solvent effect that separates the scattering objects without dissolving them. Thus, the colloidal nature due to aggregation is maintained even in systems with $60 \mathrm{wt} . \%$ toluene. $^{60}$

In addition, the effect of two model inhibitors (dodecylbenzene sulfonic acid-DBSA and noniphenol-NF) was verified by SAXS analyses. The data showed that the effect of these compounds did not affect the aggregate nanostructure (first level of organization) ${ }^{45}$ in agreement
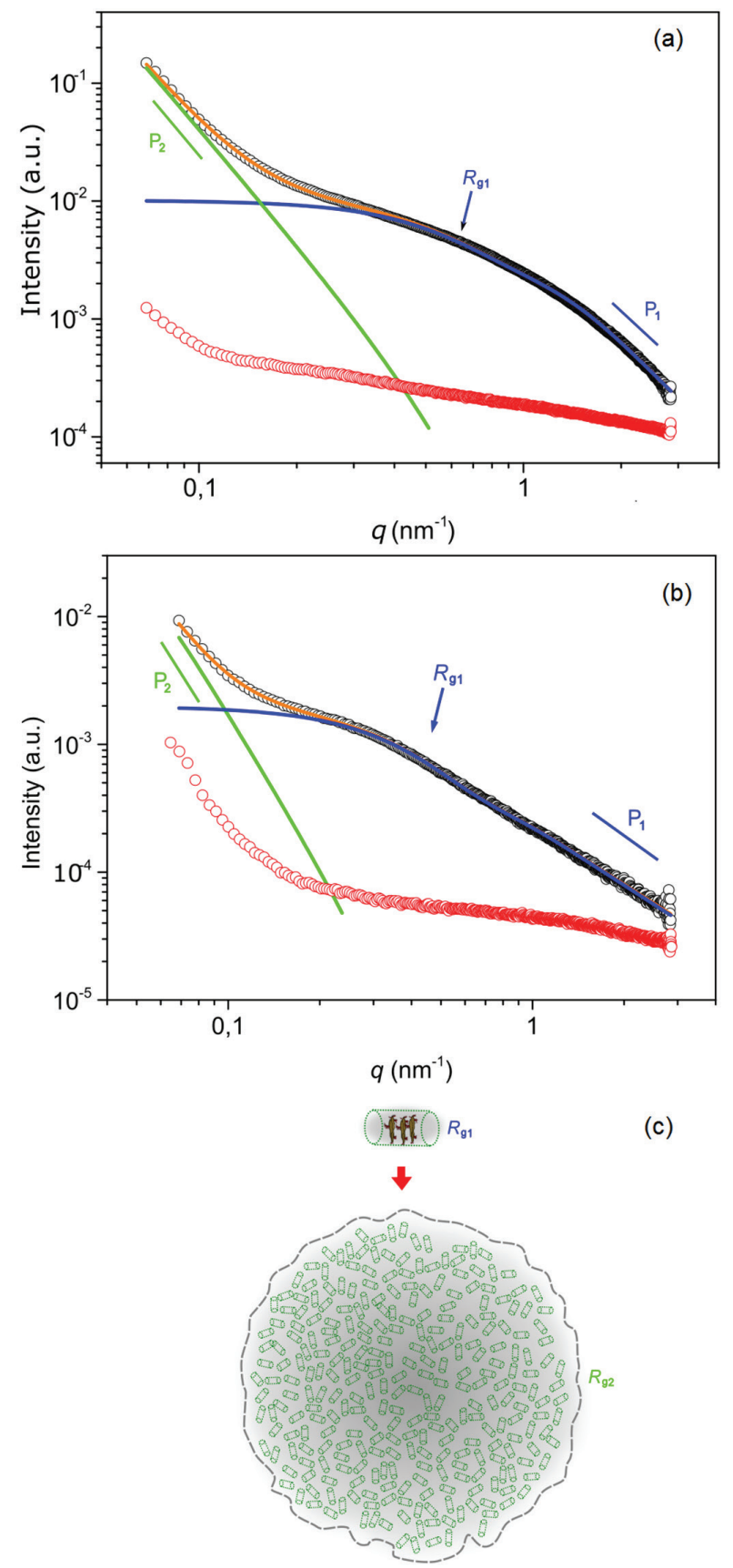

Figure 3. Small angle $\mathrm{X}$-ray scattering curves for the oils (a) OF1 and (b) OF2 (black circles) and their respective C5-maltenes (red circles) at $25{ }^{\circ} \mathrm{C}$. Unified levels are displayed in different colors: blue line corresponds to the adjustment of the first level, green line of the second level, while the orange line represents the adjustment of the two levels together. (c) Proposed schematic representation, indicating a hierarchical aggregation process. In the figure, the error bar is smaller than the symbols used for the data (reproduced from reference 45 with copyright permission 2016 from American Chemical Society).

with recent USAXS data that revealed the effect only at the next level of organization. ${ }^{58}$ In line with this observation, oil + inhibitor systems showed perturbations exclusively in submicron and micrometer size ranges through experiments conducted using a quartz crystal microbalance 

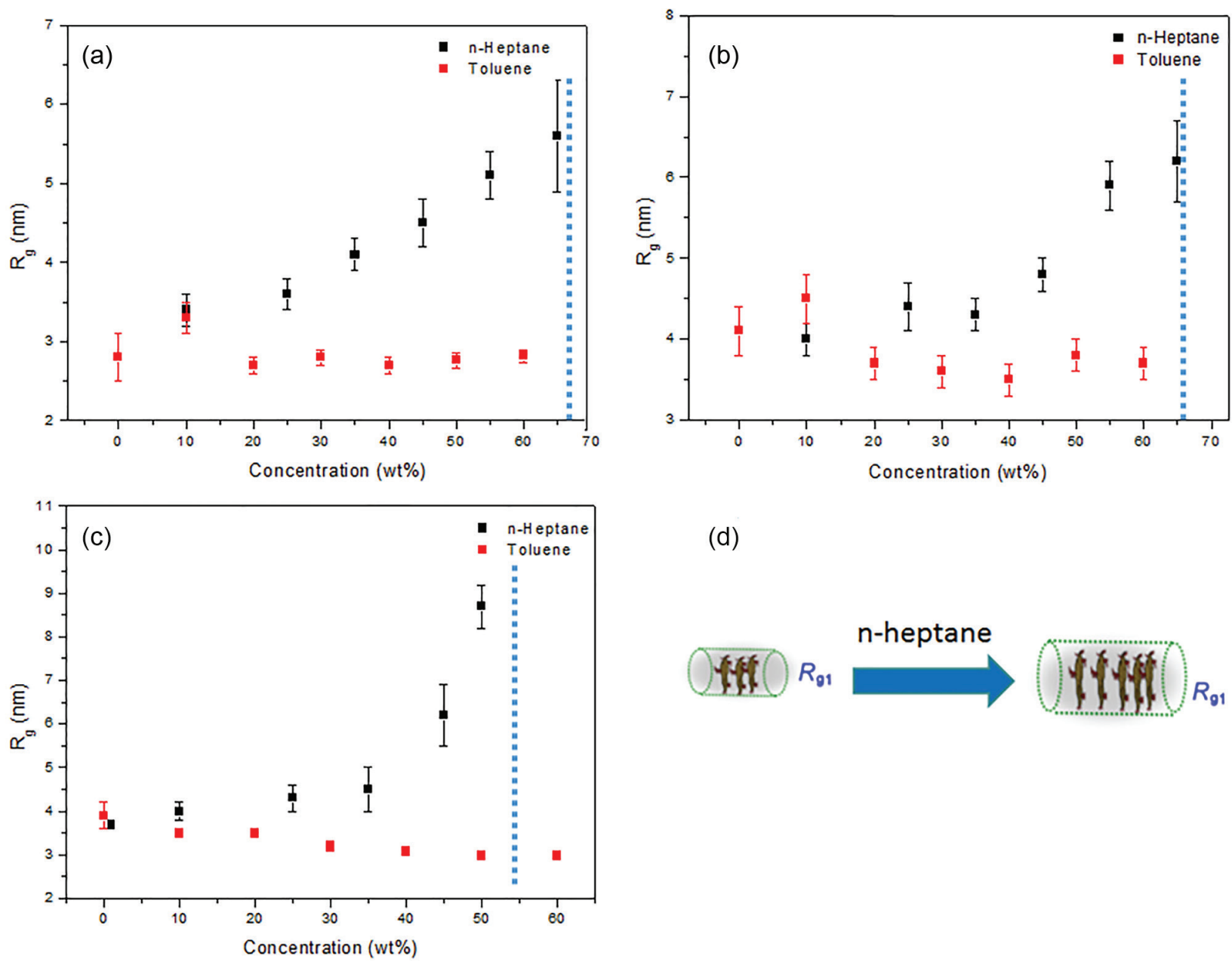

(d)

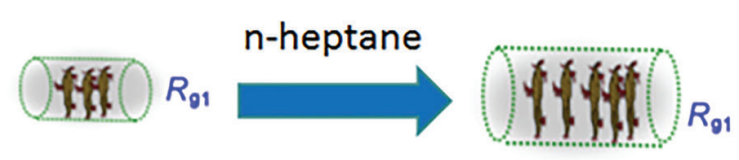

Figure 4. $R_{g 1}$ values for the first level of asphaltenes aggregation, obtained using unified equation for the oils (a) OF1, (b) OF2 and (c) OF3 at different concentrations of toluene and $n$-heptane. The error bars indicate the deviation from the fit to the model. (d) Proposed schematic representation, indicating the effect of a poor solvent. In the case of the samples with $n$-heptane, it is emphasized that the added concentration was always below the onset. In terms of mass percentage, the precipitation onset for these oils are, respectively, (a) $67 \mathrm{wt} . \%$; (b) $66 \mathrm{wt} . \%$ and (c) $55 \mathrm{wt} . \%$, indicated by a dotted blue line. ${ }^{60}$
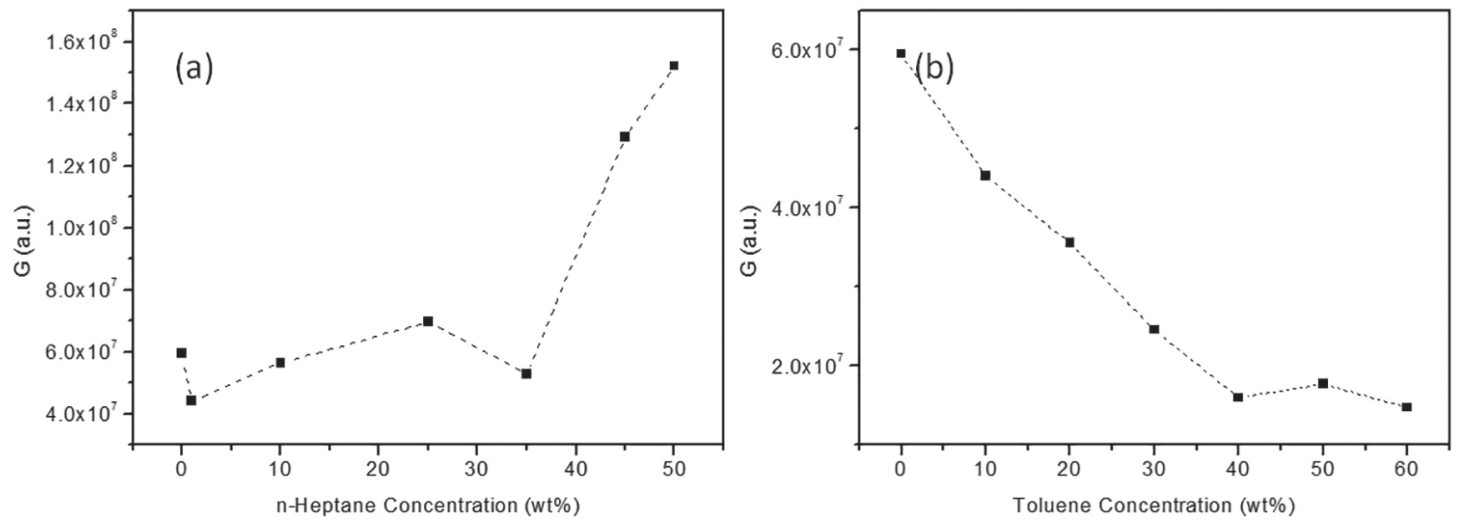

Figure 5. G values for the first organization level of the OF3 oil in which different concentrations of (a) $n$-heptane and (b) toluene were added. Dotted line in the figures represents only an eye guide. ${ }^{60}$

(QCM) and an analytical centrifuge (LUMiSizer). ${ }^{21}$ As an example, results from the LUMiSizer showed that in case of precipitation, the inhibitor acts at the submicrometric scale, avoiding the stacking of nanoaggregates in even larger structures, thus reducing the size of objects by up to an order of magnitude. ${ }^{21}$ This result indicates that probably the best starting point for the development of new inhibitors should be to assess the colloidal stability of nanoaggregates. Therefore, the sole concern with specific chemical interactions may fail. 
The results of QCM and LUMisizer techniques also showed that asphaltenes deposition on a model surface can be described by the diffusion-limited or reaction-limited aggregation mechanisms (respectively, DLA and RLA). Systems near or above the precipitation onset follow the DLA model. Reducing flocculant concentration or inhibitor addition at appropriate concentration leads to deposition behavior for RLA adjustment. ${ }^{21}$

The authors of this review were also interested in the direct visualization of these aggregates on the colloidal scale, developing a simple methodology employed in an atomic force microscope that can be accessed in detail in the reference..$^{61}$ Figure 6 shows a 3D map of a $1 \times 1 \mu \mathrm{m}$ region, indicating spherical morphology of the particles and their nature as individual units. It is possible to distinguish further that larger objects are formed by the aggregation of smaller units, whose size is $(4 \pm 1) \mathrm{nm}$, in height, ${ }^{61}$ in agreement with the values calculated by fitting the scattering curves obtained by SAXS. ${ }^{45}$ Figure 7 endorses the fact that particles at the micrometer scale are the result of subunits aggregation (roughness in topography map and border meeting of several particles in contrast phase map).

These studies with AFM showed that asphaltenes are already found as aggregates in crude oil and even in asphaltenes solutions, and that their interaction with the substrate surface is due to their colloidal and not only molecular interactions. Thus, the understanding of the surface characteristics dictates asphaltenes deposition. For example, Figure 8 shows a statistical analysis of the size distribution of aggregates on mica, gold surfaces and highly oriented pyrolytic graphite (HOPG). It is observed that, for $\mathrm{OF} 1$ oil, the effect is discrete, with the narrowing of the size distribution to the less polar surfaces. On the other hand, there is a reduction in the size of the aggregates on HOPG and gold when compared to the mica surface, in the case of OF3 oil. This fact suggests that reducing the polarity of
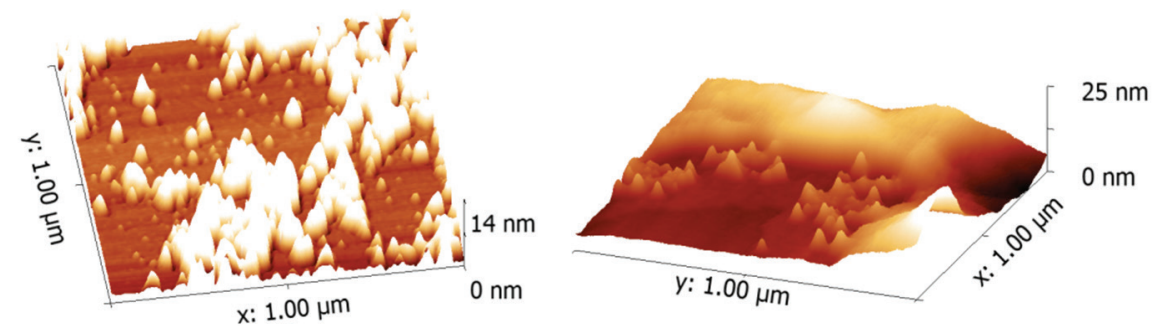

Figure 6. Three dimensional maps of OF1 oil height deposited on the surface of mica washed with toluene, subsequently. Images of two samples prepared independently. ${ }^{60}$

(a)

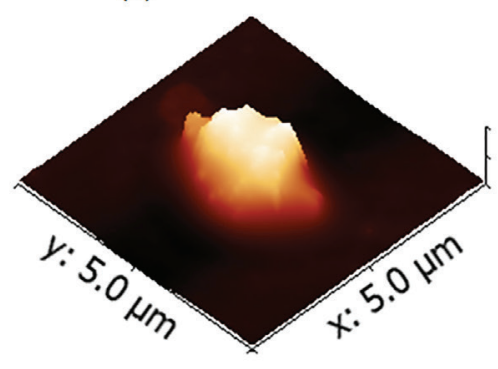

(c)

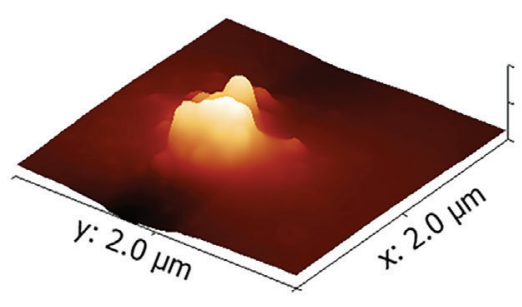

(b)

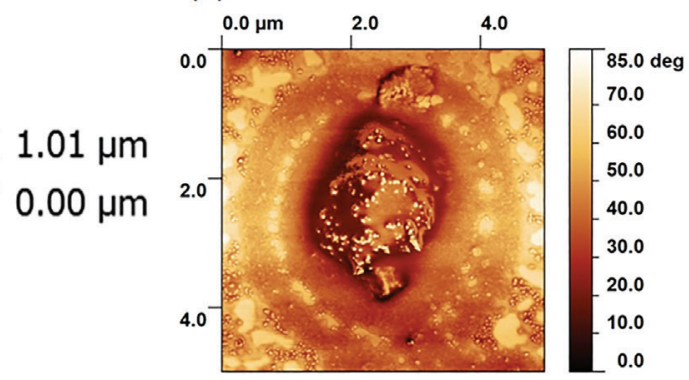

(d)

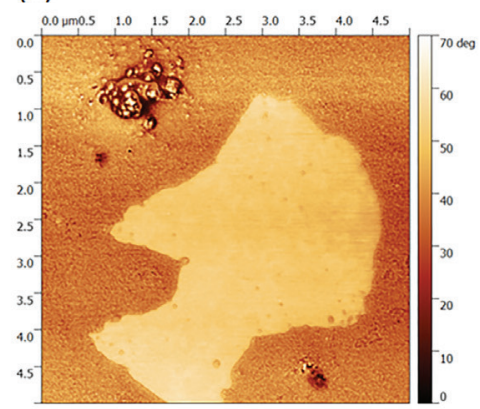

Figure 7. Maps of (a), (c): height (three-dimensional) and (b), (d): phase contrast of OF1 oil deposited on the surface of mica washed with toluene, subsequently. ${ }^{60}$ 

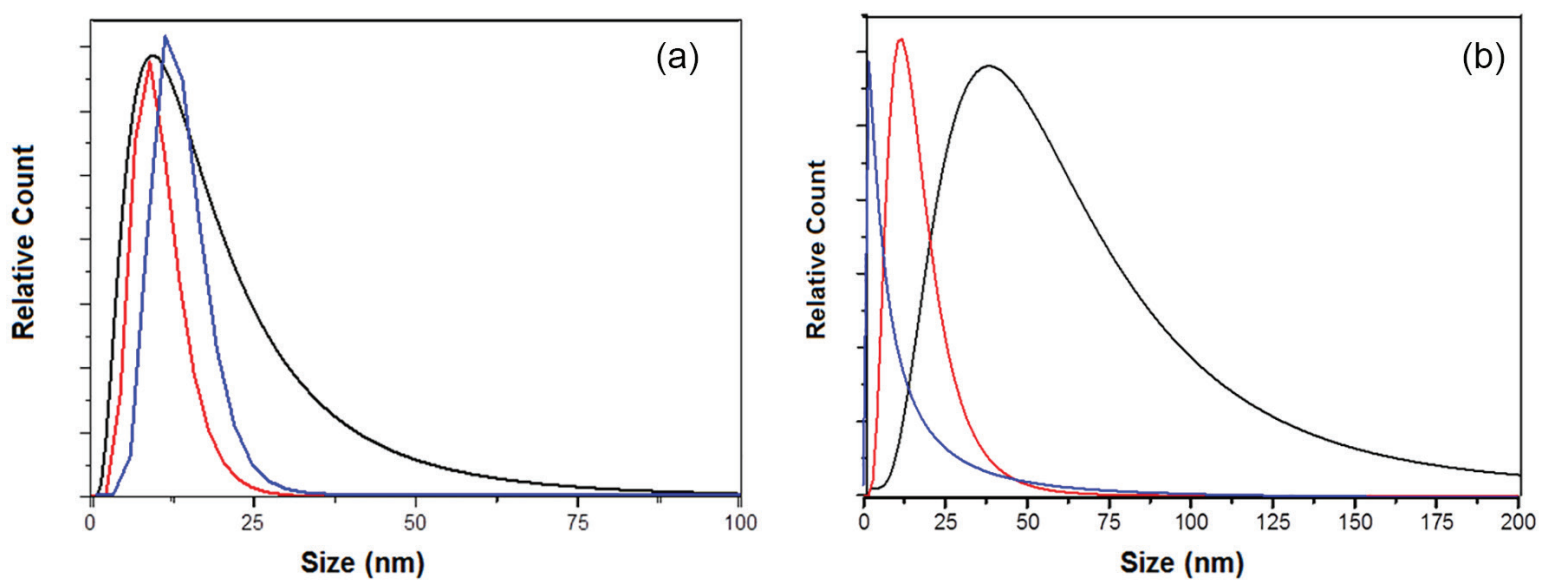

Figure 8. Overlap of the height distribution model for the asphaltenes aggregates present in the oil OF1 (a) and OF3 (b), respectively, on mica (black curve), gold (blue curve) and HOPG (red curve). In (a), median height of (16.4 \pm 0.3$) \mathrm{nm}$ for mica, $(13.38 \pm 0.06) \mathrm{nm}$ for gold and (10.08 \pm 0.02$) \mathrm{nm}$ for HOPG. In (b), median height of $(56 \pm 1) \mathrm{nm}$ for mica, $(9.7 \pm 0.1) \mathrm{nm}$ for gold and $(15.0 \pm 0.2) \mathrm{nm}$ for HOPG. Values obtained by log-normal fit. ${ }^{60}$

these surfaces reduces the tendency of asphaltenes to pile up, reducing their size..$^{60}$

In summary, we observe clear evidence of the existence of colloidal asphaltenes particles both in crude oils and asphaltenes solutions organized in at least two levels: one at nanometer and another one at submicron scales. These particles appear to grow under certain circumstances (such as the addition of flocculants) that may represent instability conditions in the field and process plants. One key aspect related to their colloidal behavior is the kinetics of their aggregation, ${ }^{21,53,54,57}$ that has been not fully considered with respect to experimental studies, regardless of sound evidences supporting its importance. Finally, there are evidences that the performance of inhibitors for asphaltenes aggregation and deposition involves colloidal interactions and not exclusively specific interactions.

\section{Implications of the Colloidal Nature of Asphaltenes Aggregation and Prospects}

The previous sections presented a brief description on how molecular interactions (represented by the thermodynamic approach) are commonly evoked to describe phenomena related to asphaltenes association and deposition. We have also reviewed a series of experimental evidences (as well as theoretical models to account for them) reported in the last years revealing that colloidal particles composed of asphaltenes are present in their solutions and in crude oils, and on how these particles play an important role in their association or interaction with surfaces, for example. These evidences emphasize the importance of considering colloidal interactions when dealing with asphaltenic related issue, including those related to the kinetics of their aggregation, that may impact even experimental determinations such as their precipitation onsets.

In this last section, we would like to re-address these issues raising a few questions that we believe are still open and that deserve to be investigated.

The first issue deals with asphaltenic composition: are the asphaltenes particles different in composition from the continuum? This would be expected considering, for example, what is observed in the fractionation of dispersed polymers taking into account that asphaltenes also display a broad molecular distribution. Mass spectrometry results on asphaltenes samples show that there is a continuum of molar mass that is present in both island and archipelago morphology. ${ }^{62}$ Even more simply, asphaltenes extraction with distinct flocculants produces asphaltenes with distinct deposition characteristics, ${ }^{55,56,63}$ that despite displaying similar molar masses, show large polarity differences that affect their phase separation pattern. ${ }^{63}$

Most importantly, if particles bear a different asphaltenes composition, would their potential for damage (for instance, deposition) be the same as that assessed for the whole or the soluble fraction? How should current models be adapted for this potential discrepancy? One key issue regarding the questions above is the elucidation of the chemical composition of asphaltenes colloidal particles in comparison with that of the whole or soluble fractions. This important but still lacking information would depend on separation of a significant amount of asphaltenes present as colloidal particles and probably subject it to a survey of chemical composition such as that performed from stateof-art mass spectrometry techniques.

Another issue is related to the role of these colloidal particles in, possibly, nucleating deposits formation. In this respect, there are studies with other nanoparticles ${ }^{64}$ (or surfaces) that indicate a significant nucleation effect. More 
specific investigations focusing on how the asphaltenes particles may interact with soluble fractions are needed, most likely using the experimental tools described in this review.

These nanoparticles are also prone to concentrate at oilwater interface and, hence, affect stability of emulsions, as has been shown in earlier investigations. ${ }^{65}$ In this respect, the elucidation of the different contributions arising from interfacially active molecules and Pickering stabilization due to asphaltenes nanoparticles should be directly investigated. There are already reports in the literature relating the formation of solid particles of resins, asphaltenes or inorganic solids in the stabilization of water-in-oil (w/o) emulsions. ${ }^{66,67}$ Chevalier et al. ${ }^{67}$ associated asphaltenes and waxes as a colloidal suspension of solid particles indicating the formation of a Pickering w/o emulsion as the mechanism to the stabilization of the water droplets. Harbottle et al. ${ }^{68}$ showed that, in addition to solid particle stabilization, the water-oil interface could be stabilized by a liquid-like microstructure, depending on asphaltene concentration, medium aromaticity and interface aging. In this case, the role of asphaltenes is to form a network film that prevents the coalescence of water droplets. Increasing the elastic modulus of the formed film increases its resistance. ${ }^{68}$

In another front, how should the development of asphaltenes inhibitors be carried out? Should it focus on molecular or colloidal interactions? How should the kinetics of colloidal processes be considered or, more fundamentally, is it relevant at all? Could we still rely on onset of precipitation values that are time-dependent? $?^{50}$

Further difficulties arise when one considers that most often other fractions such as paraffins or naphthenic acids appear in asphaltenes deposits? How do these fractions affect the structure and behavior of asphaltenes aggregates or particles? For example, in the case of naphthenic acids, there are reports of an increase in the precipitation onset of asphaltenes, indicating an increase in steric hindrance that limits aggregate growth. ${ }^{69,70}$ It is further known that asphaltenes and paraffins when mixed form a complex solid. ${ }^{71}$ In addition, the precipitation process of this complex may become irreversible. One explanation for this observation is that these compounds follow opposite phase separation processes. In the case of asphaltenes, they follow phase transition by flocculation, while paraffins separate due to crystallization..$^{72}$ Accordingly, the known bee-like structure of asphalt components (mainly asphaltene and wax) was studied by Pahlavan et al..$^{73}$ These authors showed that the $n$-paraffin wax imposes deformation on the asphaltene dimer in the wax-doped model, reducing its tendency for dimerizarion (i.e., for aggregation). Moreover, that study showed that the interaction between wax crystals is thermodynamically more stable than in a wax-doped system of asphaltenes. This suggests that waxes molecules aggregation and crystallization of wax molecules promotes bee-like structure. In the wax-asphaltene co-aggregation process, wax in found in a lamellar structure. ${ }^{73}$

Therefore, model system studies with only extracted asphaltenes fractions end up neglecting important factors in the study of asphaltenes precipitation and the assertive proposition of inhibitors.

It seems clear that, regardless of the evidence of colloid involvement, the molecular steps cannot be neglected either in the initial formation of the particles or as a source of material to interaction with the nanoparticles. It means that there is room for models based on molecular approaches such as the solubility parameter. Nonetheless, we believe that molecular and colloidal mechanisms should be better integrated, perhaps even indicating more clearly in the steps of the Yen-Mullins model which laws control each step, and which, if any exists, would be the determining step for the problem under investigation (most commonly deposition).

Based on the studies and evidences reviewed here, we suggest an integrated scheme for the role of molecular and colloidal steps in the comprehensive aggregation model (Figure 9). ${ }^{60}$ The vertical dotted line separates the molecular from the colloidal regime. According to this scheme, interactions leading to the formation of the (nano) aggregates are governed by the molecular model. Sequential growth of these aggregates by assembling smaller aggregates and/or other molecules can result in larger flakes that sediment, forming a precipitate. As indicated in Figure 9, the nomenclature used, whether aggregate, flake or precipitate is open, but it should be clear that, at this stage, phase separation already occurred, and that these entities are no longer dissolved, hence not being governed by equilibrium thermodynamics involved in molecular interactions. For this regime, the experimental evidences suggest a major role of colloidal interactions over specific interactions. ${ }^{60}$ According to this picture, the transition between regimes is not associated with the size of the aggregates, but with their nature, reason why we do not believe setting size limits for these entities would solve the situation.

As stated above, this mechanism is not only complex for involving interactions of different nature (hence governed by different laws) but can increase in complexity if considering participation of other compounds represented by other oil fractions (such as resins, naphtenic acids or waxes/paraffins).

Therefore, the aim of the proposals advanced in this review is not towards simplification of this complex mechanism, but for a clearer discrimination of the events under scrutiny that would ease communication amongst the players involved in their investigation. Perhaps, as a bonus, these more direct analyses would facilitate advances 
towards understanding of the different steps involved in asphaltenic deposition issues, including development and assessment of additives for its mitigation and inhibition.

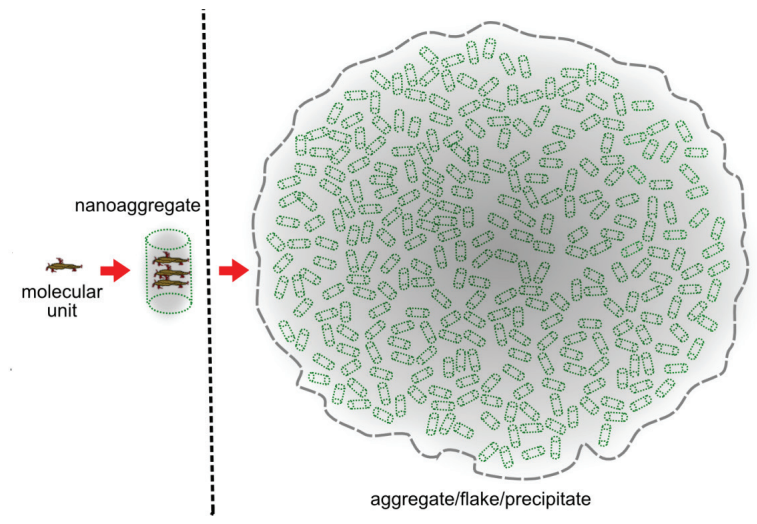

Figure 9. Scheme indicating the aggregation model supported by the data presented in this review. Dotted line indicates the transition between colloidal and molecular regimes. ${ }^{60}$

\section{Acknowledgments}

The authors thank the Brazilian Agencies FAPESP (2015/25406-5) and CNPq for support to this work through a PhD scholarship (CNPq 140372/2015-6) to L. B. S. B and productivity grant to $\mathrm{W}$. $\mathrm{L}$.

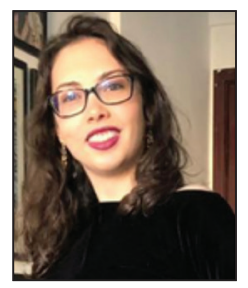

Lia Beraldo da Silveira Balestrin obtained her BSc (2012), MSc (2015) and PhD (2019) in Chemistry from the University of Campinas, Brazil. During her training, she studied different aspects of surface and colloid Physical Chemistry, dealing initially with charged surfaces and during her PhD work she studied asphaltenes aggregation, employing experimental techniques such as small angle X-ray scattering and atomic force microscopy to characterize asphaltenes aggregates in their colloid aspects. She is currently a researcher at Oxiteno Company, working in the Oil \& Gas section.

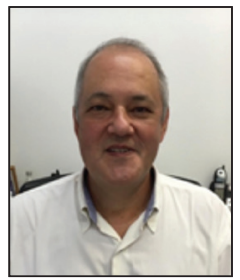

Watson Loh obtained his BSc in Chemistry from the Federal University of Rio Grande do Sul (1986) and his $P h D$ in Chemistry from the University of Campinas (1992). He is a Professor at the Institute of Chemistry of the University of Campinas, where since 1994 he leads a research group devoted to studying different aspects of polymer and surfactant colloids (including petroleum colloids) using techniques such as light and $X$-ray scattering, scanning and titration calorimetry among others. He is associated with Advisory Boards of different international conferences (IACIS, SIS, Polyelectrolytes, AutoOrg) and Journals (Chem. Sci., J. Chem. Thermodyn., ACS Omega) in these fields and is currently an Associate Editor for the Journal of Surfactants and Detergents. He is a member of the São Paulo State Academy of Sciences and a Fellow of the Royal Society of Chemistry.

\section{References}

1. Akmaz, S.; Iscan, O.; Gurkaynak, M. A.; Yasar, M.; Pet. Sci. Technol. 2011, 29, 160

2. Fan, T.; Wang, J.; Buckley, J. S.; In SPE/DOE Improved Oil Recovery Symposium; Society of Petroleum Engineers: Tulsa, 2002, 883, SPE 75228.

3. Manual on Hydrocarbon Analysis, $6^{\text {th }}$ ed.; Drews, A., ed.; American Society for Testing and Materials: West Conshohocken, 2008, p. 311.

4. Zohuriaan-Mehr, M. J.; Omidian, H.; J. Macromol. Sci., Rev. Macromol. Chem. Phys. 2000, 40, 23.

5. Mullins, O. C.; Annu. Rev. Anal. Chem. 2011, 4, 393.

6. Nikooyeh, K.; Bagheri, S. R.; Shaw, J. M.; Energy Fuels 2012, 26,1756

7. Loh, W.; Mohamed, R. S.; dos Santos, R. G. In Encyclopedia of Surface and Colloid Science; Somasundaram, P., ed.; Taylor \& Francis: New York, USA, 2007.

8. Goual, L. In Crude Oil Emulsions-Composition Stability and Characterization; Abdel-Raouf, P. M. E.-S., ed.; InTech: Rijeka, Croatia, 2012, 27.

9. Speight, J. G.; Oil Gas Sci. Technol. 2004, 59, 467.

10. Asphaltene Deposition - Fundamentals, Prediction, Prevention, and Remediation; Vargas, F. M.; Tavakkoli, M., eds.; CRC Press: New York, 2018

11. Mullins, O. C.; Sabbah, H.; Pomerantz, A. E.; Barre, L.; Andrews, A. B.; Ruiz-Morales, Y.; Mostowfi, F.; McFarlane, R.; Goual, L.; Lepkowicz, R.; Cooper, T.; Orbulescu, J.; Leblanc, R. M.; Edwards, J.; Zare, R. N.; Eyssautier, J.; Barré, L.; Energy Fuels 2012, 26, 3986.

12. Gray, M. R.; Tykwinski, R. R.; Stryker, J. M.; Tan, X.; Energy Fuels 2011, 25, 3125.

13. Diner, C.; Scott, D. E.; Tykwinski, R. R.; Gray, M. R.; Stryker, J. M.; J. Org. Chem. 2015, 80, 1719.

14. Chacón-Patiño, M. L.; Rowland, S. M.; Rodgers, R. P.; Energy Fuels 2018, 32, 9106.

15. Chang, C.-L.; Fogler, H. S.; Langmuir 1994, 10, 1749.

16. Argillier, J.-F.; Coustet, C.; Henaut, I.; In SPE International Thermal Operations and Heavy Oil Symposium and International Horizontal Well Technology Conference; Society of Petroleum Engineers: Calgary, 2002, SPE 79496-MS.

17. Spiecker, P. M.; Gawrys, K. L.; Kilpatrick, P. K.; J. Colloid Interface Sci. 2003, 267, 178. 
18. Wang, J.; Buckley, J. S.; Energy Fuels 2003, 17, 1445.

19. Kokal, S. L.; Sayegh, S. G.; In Middle East Oil Show; Society of Petroleum Engineers: Bahrain, 1995, SPE 29787-MS.

20. Alrashidi, H.; Afra, S.; Nasr-El-Din, H. A.; J. Pet. Sci. Eng. 2019, 172, 724.

21. Balestrin, L. B. D. S.; Francisco, R. D.; Bertran, C. A.; Cardoso, M. B.; Loh, W.; Energy Fuels 2019, 33, 4748.

22. Subramanian, S.; Simon, S.; Sjöblom, J.; J. Dispersion Sci. Technol. 2016, 37, 1027.

23. Sato, T.; Araki, S.; Morimoto, M.; Tanaka, R.; Yamamoto, H.; Energy Fuels 2014, 28, 891.

24. Rogel, E.; Ovalles, C.; Bake, K. D.; Zuo, J. Y.; Dumont, H.; Pomerantz, A. E.; Mullins, O. C.; Energy Fuels 2016, 30, 9132.

25. Rogel, E.; Colloids Surf., A 1995, 104, 85.

26. Rogel, E.; Langmuir 2002, 18, 1928.

27. Rogel, E.; Energy Fuels 2008, 22, 3922.

28. Novaki, L. P.; Lira, R.; Kwon, M. M. N.; de Oliveira, M. C. K.; Meireles, F. A.; Gonzalez, G.; El Seoud, O. A.; Energy Fuels 2018, 32, 3281.

29. Ortega-Rodríguez, A.; Cruz, S. A.; Gil-Villegas, A.; GuevaraRodríguez, F.; Lira-Galeana, C.; Energy Fuels 2003, 17, 1100.

30. Hashmi, S. M.; Firoozabadi, A.; Energy Fuels 2012, 26, 4438.

31. Nabzar, L. T. C. A.; Aguiléra, M. E.; Oil Gas Sci. Technol. 2008, 63, 21.

32. Sheu, E. Y.; Energy Fuels 2002, 16, 74.

33. Nellensteyn, F. J.; J. Inst. Pet. Technol. 1924, 10, 311.

34. Moir, M. E.; ACS Symp. Ser. 2018, 1282, 3.

35. Pfeiffer, J. P.; Saal, R. N.; J. Phys. Chem. 1940, 44, 139.

36. Alkafeef, S. A.; In SPE International Symposium on Oilfield Chemistry; Society of Petroleum Engineers: Houston, 2001, SPE 65018-MS.

37. Dickie, J. P.; Yen, T. F.; Anal. Chem. 1967, 39, 1847.

38. Yen, T. F.; Erdman, J. G.; Pollack, S. S.; Anal. Chem. 1961, 33, 1587.

39. Schuler, B.; Meyer, G.; Peña, D.; Mullins, O. C.; Gross, L.; J. Am. Chem. Soc. 2015, 137, 9870.

40. Sedghi, M.; Goual, L.; Energy Fuels 2010, 24, 2275.

41. Hoepfner, M. P.; Fogler, H. S.; Langmuir 2013, 29, 15423.

42. Hoepfner, M. P.; Fávero, C. V. B.; Haji-Akbari, N.; Fogler, H. S.; Langmuir 2013, 29, 8799.

43. Eyssautier, J.; Levitz, P.; Espinat, D.; Jestin, J.; Grillo, I.; Barré, L.; J. Phys. Chem. B 2011, 115, 6827.

44. Sirota, E. B.; Energy Fuels 2005, 19, 1290.

45. Padula, L.; Balestrin, L. B. S.; Rocha, N. D. O.; de Carvalho, C. H. M.; Westfahl, H.; Cardoso, M. B.; Sabadini, E.; Loh, W.; Energy Fuels 2016, 30, 3644.

46. Agrawala, M.; Yarranton, H. W.; Ind. Eng. Chem. Res. 2001, 40, 4664.

47. Dechaine, G. P.; Gray, M. R.; Energy Fuels 2011, 25, 509.

48. Zhang, H. T.; Li, R.; Yang, Z.; Yin, C.-X.; Gray, M. R.; Bohne, C.; Photochem. Photobiol. Sci. 2014, 13, 917.
49. Rapacioli, M.; Calvo, F.; Spiegelman, F.; Joblin, C.; Wales, D. J.; J. Phys. Chem. A 2005, 109, 2487.

50. Saville, P. M.; Sevick, E. M.; Langmuir 1998, 14, 3137.

51. Zahl, P.; Zhang, Y.; Energy Fuels 2019, 33, 4775.

52. Evdokimov, I. N.; Energy Fuels 2019, 33, 8440.

53. Maqbool, T.; Balgoa, A. T.; Fogler, H. S.; Energy Fuels 2009, 23, 3681 .

54. Vilas Bôas Fávero, C.; Maqbool, T.; Hoepfner, M.; Haji-Akbari, N.; Fogler, H. S.; Adv. Colloid Interface Sci. 2016, 244, 267.

55. Abudu, A.; Goual, L.; Energy Fuels 2009, 23, 1237.

56. Yang, G.; Chen, T.; Zhao, J.; Yu, D.; Liu, F.; Wang, D.; Fan, M.; Chen, W.; Zhang, J.; Yang, H.; Wang, J.; Energy Fuels 2015 , 29, 4272.

57. Zahabi, A.; Gray, M. R.; Dabros, T.; Energy Fuels 2012, 26, 1009.

58. Ismail, M.; Yang, Y.; Chaisoontornyotin, W.; Ovalles, C.; Rogel, E.; Moir, M. E.; Hoepfner, M. P.; Energy Fuels 2018, 33, 3681.

59. Beaucage, G.; J. Appl. Crystallogr. 1995, $28,717$.

60. Balestrin, L. B. S.: Caracterização Coloidal de Asfaltenos em Petróleo e Efeito de Aditivos sobre seu Mecanismo de Deposição; $\mathrm{PhD}$ thesis, University of Campinas, 2019, available at http://repositorio.unicamp.br/handle/REPOSIP/335392.

61. Balestrin, L. B. S.; Cardoso, M. B.; Loh, W.; Energy Fuels 2017, 31, 3738.

62. Chacón-Patiño, M. L.; Rowland, S. M.; Rodgers, R. P.; Energy Fuels 2018, 32, 314.

63. Ferreira, S. R.; Barreira, F. R.; Spinelli, L. S.; Leal, K. Z.; Seidl, P.; Lucas, E. F.; Quim. Nova 2016, 39, 26.

64. Nassar, N. N.; Hassan, A.; Pereira-Almao, P.; Energy Fuels 2011, 25, 1017.

65. Ramos, A. C. S.; Haraguchi, L.; Notrispe, F. R.; Loh, W.; Mohamed, R. S.; J. Pet. Sci. Eng. 2001, 32, 201.

66. Wang, X.; Alvarado, V.; In SPE Annual Technical Conference and Exhibition; Society of Petroleum Engineers: Denver, 2008, SPE 115941-MS.

67. Chevalier, Y.; Bolzinger, M. A.; Colloids Surf., A 2013, 439, 23.

68. Harbottle, D.; Chen, Q.; Moorthy, K.; Wang, L.; Xu, S.; Liu, Q.; Sjoblom, J.; Xu, Z.; Langmuir 2014, 30, 6730.

69. Heaps, D. T.; Madasu, P. K.; Magers, D. H.; Buchanan, J. P.; Energy Fuels 2012, 26, 1862.

70. Carbonezi, C. A.; Almeida, L. C. D.; Araujo, B. C.; Lucas, E. F.; González, G.; Energy Fuels 2009, 23, 1249.

71. Garcia, M. C.; Carbognani, L.; Energy Fuels 2001, 15, 1021.

72. Leontaritis, K. J.; Fuel Sci. Technol. Int. 1996, 14, 13.

73. Pahlavan, F.; Mousavi, M.; Hung, A.; Fini, E. H.; Phys. Chem. Chem. Phys. 2016, 18, 8840.

Submitted: September 2, 2019

Published online: November 8, 2019 